\title{
HORMONAL AND METABOLIC RESPONSES TO MODERATE AND HEAVY RESISTANCE EXERCISE LOADS IN MALE SUBJECTS
}

\author{
El-Ayadi D. El-Abed*and Shousha S. ** \\ *GIT (liver and metabolic diseases), Faculty of Medicine, Zawia Univ, Libya \\ **Physiology, Nutr. Dept., Fac. Pub. Health, Zawia Univ., Libya
}

\begin{abstract}
Forty healthy male subjects as volunteers participated in two different exercise load protocols (moderate and heavy loads), 20 subjects for each. The exercise protocols were carried out in the morning after fasting overnight, five times a week for 30 minutes a day for four weeks. Blood samples were withdrawn before the start and immediately after finishing the exercise protocols, from the antecubital vein, and the obtained plasma were kept at $-20{ }^{\circ} \mathrm{C}$ until analysis. The hormone level of growth hormone (GH), testosterone, cortisol, insulin, $\mathrm{T}_{3}$ and $\mathrm{T}_{4}$, were determined by RIA technique, while the plasma concentrations of glucose, non-esterified free fatty acids (NEFFAs), triglycerides (TG) and total protein (TP) were measured biochemically. The results revealed a significant increase in the hormone levels of $\mathrm{GH}$, testosterone, cortisol, insulin and $\mathrm{T}_{4}$; ; and a significant increase in the concentration of NEFFAs and TG, while the plasma $\mathrm{T}_{3}$ levels and plasma glucose concentration showed a significant decrease in both exercise load protocols. Moreover, the $T_{3}$ levels indicated a positive correlation coefficient (r) with glucose concentration, and a negative (r) with both NEFFAs and TG. On the other hand, $\mathrm{T}_{4}$, insulin and cortisol revealed a negative (r) with glucose and a positive (r) with both NEFFAs and TG. The results reported here were similar to a great extent to that reported in starvation. Thus, it would clearly appear that the exercise protocols can be considered as a good regimen program.
\end{abstract}

Keywords: Exercise loads, growth hormone, lactate, cortisol, NEFAs

\section{INTRODUCTION}

E ndurance training, as a complex process, involves not only cardiovascular and muscular adaptations to exercise, including diminished heart rate (HR), at rest and during submaximal exercise, increased maximal oxygen uptake $\left(\mathrm{VO}_{2} \mathrm{max}\right)$, decreased blood lactate accumulation with a subsequent shift of the anaerobic threshold towards higher workloads, but also changes endocrine responses to exercise (1). Similarly to hypoxia, acute or chronic exercise significantly modulates hormonal appetite regulation (2). Acute exercise transiently suppresses appetite, while chronic exercise training typically results in an augmented appetite leading to a restoration of the exerciseinduced energy imbalance (3). While combining hypoxia and exercise is mostly used by athletes aiming to improve performance (4), it is gaining popularity as a novel modality for treatment of obesity with related co-morbidities (5). It has been suggested that hypoxia and exercise synergistically enhance metabolic health, since both individually up-regulate glucose, glycolysis and satiety related genes through hypoxia-inducible factor-1 stabilization (6). However, Fatouros et al (7) showed that resistance exercise might represent an effective approach for weight management and metabolic control in overweight elderly individuals.
In terms of the underlying determinants of exercise strength and power, three stimuli (mechanical, hormonal and metabolic) are thought important. In addition, the mechanical stimulus (e.g. high tension, stretch, time under tension) afford by resistance exercise is thought to be the most important stimuli for training - induced adaptations to occur $(\mathbf{8})$.

Endocrine responses to resistance exercise are thought to be important in the development of muscle strength and power (9). The interaction between the anabolic (e.g. testosterone and growth hormones) and catabolic (e.g. cortisol) hormones regulate the balance between protein synthesis and degradation (10). Resistance exercise is known to elicit acute and chronic changes in blood hormone levels, thereby mediating the long-term process of muscle tissue growth (10). As different training protocols produce differential hormonal responses, program design plays an important role in modulating muscle growth (11).

The metabolic response to resistance exercise may also be important for muscle strength and to a lesser extent power development (12). The stimulus for strength and power adaptations might result from changes in the level of circulating metabolites (e.g. lactate, creatine kinase, glycogen, etc.) as a consequence of resistance exercise and the effect of 
these metabolites upon the muscle environment. Such changes might contribute to the training stimulus by stimulating greater anabolic hormone release (13). Thus, the present study was designed to determine how quickly the moderate and heavy exercise loads program evoke the hormonal changes in previously sedentary men and if so whether these hormonal responses to exercise correlate with other training-induced metabolic changes.

\section{MATERIALS AND METHODS}

Forty male subjects as volunteers were participated in the present study. Their average age (yrs), weight $(\mathrm{Kg})$ and height $(\mathrm{cm})$ were $19 \pm 0.62$, $64 \pm 4$ and $170 \pm 8$ respectively. They were divided into two groups, 20 subjects for each. The first group performed the moderate exercise load protocol while the second group carried out the heavy exercise load protocol. All subjects were considered to be in good health after a comprehensive medical evaluation including history and physical examination. None was using any medications during the course of their study or reported any previous history of smoking.

\section{Exercise Protocols:}

In the two exercise protocols, all subjects performed their own specific exercise protocol in the morning after fasting overnight. The order of the exercise protocols involved cycling on a stationary bicycle ergometer (Hellige, Dynavit Meditronic M 400). After a period of load less pedaling for 3 minutes, the power was increased stepwise every 2 minutes by 15 watt from an initial load of 60 watt (protocol 1: moderate exercise) and by 30 watt from an initial load of 120 watt (protocol 2: heavy load), until the subject was unable to maintain a pedaling rate of $70 /$ minute. The exercise protocols were done five times a week for 30 minutes a day for four weeks.

\section{Blood sampling and analysis:}

In the first day, before the start of exercise protocol and in the last day immediately after finishing the exercise protocols, blood samples were withdrawn from the antecubital vien in heparinized polypropylene tubes. Blood samples were centrifuged using cooling centrifuge $\left(4^{\circ} \mathrm{C}\right)$ for 15 minutes at $3000 \mathrm{rpm}$. The obtained plasma samples for the hormonal analyses were kept frozen at $-20^{\circ}$ $\mathrm{C}$ until assayed. The assays of serum cortisol and testosterone were performed by radioimmunoassays (RIAs) using reagent kits from Farmos Diagnostica (Turku, Finland). Plasma growth hormone concentrations were measured using kits of
Pharmacia Diagnostics (Uppsala, Sweden). The sensitivity of the cortical assay was $0.05 \mu \mathrm{mol} \cdot \mathrm{L}-1$, and the coefficient of intra-assay variation was $4.0 \%$. The respective values were $0.36 \mathrm{nmol} \cdot \mathrm{L}-1$ and $6.5 \%$ for the testosterone assay, and $0.2 \mu \mathrm{g} \cdot \mathrm{L}-1$ and $2.5-5.1 \%$ for the GH assay. All the assays were carried out according to the instructions of the manufacturers. All samples for each test subject were analyzed in the same assay for each hormone. The plasma levels of $T_{3}, T_{4}$, testosterone and insulin determined according to Abraham (14), Kosaka et al. (15), Ishikawa et al (16) and Abraham et al (17) orderly while plasma glucose, NEFFAs, TG and TP concentrations were measured biochemically according to Cooper and Danial (18), Falhot and Falhot (19), Wieland (20) and Weichselbaum (21) respectively. The results were statistically analyzed using student t-test to indicate the significance of differences in the means of the data and in the correlation coefficient (r) according to Snedecor and Cochran (22).

\section{RESULTS AND DISCUSSION}

The primary finding of the present study was that both moderate and heavy exercise load protocols led to a significant increase in cortisol, $\mathrm{GH}$, and lactate. These increases were represented by 42.7 and 56 folds in $\mathrm{GH} ; 71 \%$ and $44.7 \%$ in cortisol; and 3.5 and 5.3 folds in lactate as shown tables 1, 2, 3 and 4. These results were in good agreement with a similar study of Kraemer et al (23) who found a significant increase in plasma cortisol levels in women in response to heavy resistance exercise protocols, and they indicated that hormonal and metabolic blood variables varied in response to different heavy resistance exercise protocols. Moreover, the increased cortisol response in the current study may be partially explained by Burgess et al (24), who found that highly trained athletes have increased corticotrophic-releasing factor along with mild hypercortisolism. In the earlier studies of Schwarz and Kinderman (25), it was observed that short-term physical exercise can increase serum cortisol levels. Furthermore, Hakkinen and Pakarinen (26) showed that heavy resistance exercise caused acute endogenous cortisol responses that can differ depending upon the type and/or the magnitude of stress of the exercise protocol utilized.

Low- and moderate-intensity resistance exercise resulted in a substantially greater metabolic stress than high-intensity resistance exercise based on greater lactate and cortisol responses (27). Protocols 
eliciting the greatest cortisol response also elicit the greatest acute growth hormone and lactate response (28). The increase in lactate also coincides with a cortisol increase (28), suggesting that lactate may be a factor contributing to the cortisol increase seen with resistance exercise. It appears that cortisol responses to resistance exercise depend on metabolic requirements, which appear to be higher in the low- to moderate-intensity protocols, based on the lactate response and energy cost of these protocols.

Regarding to testosterone, it revealed a significant increase only in the heavy exercise load. These results were in good agreement with the results of Linnamo et al (29). They showed that heavy resistance exercise is known to lead to acute increases in serum $\mathrm{GH}$ and testosterone concentrations. Since GH and testosterone are anabolic hormones, the acute exercise-induced response during heavy exercise loads might play an important role in the long-term anabolic adaptation processes related to muscle hypertrophy and maximal strength development (29). Furthermore, Kramer and Ratamess (28) have summarized that protocols high in volume do tend to produce acute hormonal elevations in testosterone (as well as cortisol and growth hormone).

Fatouros et al (7), detected a significant increases in plasma concentration of lactate, glucose, NEFAs, and glycerol after exercise and returned to baseline thereafter in all groups. However, the results of the current endeavor showed a significant increase of $36 \%$ and $52 \%$ in plasma insulin levels in both groups performing moderate and heavy load exercise protocols respectively, while the glucose concentration showed a significant decrease of $25 \%$ and $27 \%$ in the same orderly groups. In addition, a negative (r) $(-0.88$ and - 0.41) between insulin levels and glucose concentrations were observed in both groups. Dela et al (30) have shown that in humans, physical training increases the sensitivity and responsiveness of insulin-mediated glucose uptake in skeletal muscle by local mechanisms and the glucose storage as well as glycolysis are increased after training. In the study of king et al (31) glucose disposal was significantly decreased at submaximal insulin levels but not at a maximally effective level after ten days without exercise. These findings provide evidence that the decrease in sensitivity to insulin rather than to a decrease in insulin responsiveness. Furthermore, the results of King et al (31) provide further evidence that the primary mechanism by which exercise results in an increased action involved an increase in sensitivity to insulin. The results of the present study revealed a decrease of $21 \%$ and $26 \%$ in the plasma $\mathrm{T}_{3}$, while $\mathrm{T}_{4}$ showed an increase of $37 \%$ and $62 \%$ in both exercise loads orderly. In addition, the (r) values of $\mathrm{T}_{3}$ with glucose, NEFFAs and TG were respectively as following: (0.72 and 0.76), (-0.82 and -0.93$)$ and $(-0.89$ and -0.94$)$. while the $(\mathrm{r})$ values of $\mathrm{T}_{4}$ with the same parameters orderly in both exercise protocols were $(-0.73$ and -0.79$),(0.78$ and 0.86$)$ and $(0.81$ and 0.92). However, Ahlborg et al (32) stated that exercise can be considered a form of acute or accelerated starvation and since Vagenakis et al (33) indicated that serum $\mathrm{T}_{3}$ decreases during starvation, thus it is not surprising that similar changes in thyroid hormones occur during exercise. The results of the current study were consistent with that of O'Connell et al (34) who found a significant decrease in $\mathrm{T}_{3}$ and a significant increase in $\mathrm{T}_{4}$. In addition, $\mathrm{T}_{3}$ correlated with NEFFAs and glucose, but when glucose was infused, these correlations decreased. They indicated that since moderate prolonged exercise induced a state of acute starvation, it was concluded that the changes in the peripheral thyroid hormones during exercise were similar to those found in starvation. Moreover, the temporal changes of plasma $\mathrm{rT}_{3}, \mathrm{NEFFAs}$ and glucose during exercise, suggested a relationship between thyroid hormones and the uptake and utilization of NEFFAs and glucose or the mixture of these body fuels. The present study indicated a significant increase of (157\% and 205\%) and (117\% and $134 \%$ ) in both plasma NEFFAs and TG in both exercise protocols orderly. These results agreed the results of Romijn et al (35) who observed that basal resting lipid kinetics in endurance-trained athletes were shifted toward increased mobilization and oxidation of fat. Moreover, the basal rate of triglyceride-fatty acid cycling was two to four fold higher in trained athletes. Furthermore, Knapic et al (36) showed that low-intensive exercise can be maintained after several days without food by a substantial change in fuel utilization involving increased fat utilization, muscle glycogen sparing and reduced glucose flux. Based up on the present study and the antecedent literature, it is proposed that exercise protocols may be undertaken as a therapy for lean people or as a regimen program. 
Table (1): Plasma concentrations of $\mathrm{GH}$, testosterone, cortisol, insulin, $\mathrm{T}_{3}$ and $\mathrm{T}_{4}$, ; and the type of change in these parameters in subjects performed moderate load exercise protocol.

\begin{tabular}{lccc}
\hline & Pre-exercise & Post-exercise & Type of Change \\
\hline GH $\boldsymbol{\mu m o l} / \mathbf{L}$ & 0.26 & 11.1 & $(+) 42.7$ \\
\hline Testosterone nmol/L & 8.52 & 9.38 & $(+) 8.4 \%$ \\
\hline Cortisol $\boldsymbol{\mu g} / \mathbf{D L}$ & $83.6 \pm 4.6$ & $143 \pm 6.8^{*}$ & $(+) 71$ \\
\hline Insulin $\boldsymbol{\mu} \mathbf{U} / \mathbf{L}$ & $7.3 \pm 0.42$ & $9.96 \pm 0.32^{*}$ & $(+) 36$ \\
\hline T3 ng/DL & $162 \pm 11.2$ & $128 \pm 4.8^{*}$ & $(-) 21$ \\
\hline T4 $\boldsymbol{\mu g} / \mathbf{D L}$ & $7.46 \pm 0.8$ & $10.2 \pm 0.6^{*}$ & \\
\hline Mean \pm SE & $*$ Significantly at $\mathrm{P} \leq 0.05$ & \\
$* *$ Significant at $\mathrm{P} \leq 0.01$ & $(+)=$ increase $(-)$ decrease &
\end{tabular}

Table (2): Plasma concentrations of GH, testosterone, cortisol, insulin, $\mathrm{T}_{3}$ and $\mathrm{T}_{4}$, ; and the type of change in these parameters in subjects performed heavy load exercise protocol.

\begin{tabular}{|c|c|c|c|}
\hline & Pre-exercise & Post-exercise & Type of Change \\
\hline $\mathrm{GH} \mu \mathrm{mol} / \mathrm{L}$ & $0.29 \pm 0.08$ & $16.3 \pm 1.8^{* *}$ & (+) 56 folds \\
\hline Testosterone nmol/L & $7.81 \pm 1.1$ & $26.3 \pm 2.24 * *$ & (+) 3.4 folds \\
\hline Cortisol $\mu \mathrm{g} / \mathrm{DL}$ & $91.2 \pm 4.6$ & $132 \pm 6.8^{*}$ & $(+) 44.7$ \\
\hline Insulin $\mu \mathrm{U} / \mathrm{L}$ & $6.8 \pm 0.42$ & $10.1 \pm 0.32^{*}$ & (+) 36 \\
\hline T3 ng/DL & $156 \pm 11.2$ & $112 \pm 4.8^{*}$ & $(-) 21$ \\
\hline $\mathrm{T} 4 \mu \mathrm{g} / \mathrm{DL}$ & $7.22 \pm 0.8$ & $9.86 \pm 0.6^{*}$ & (+) 37 \\
\hline & $\begin{array}{l} \pm \mathrm{SE} \\
\text { ficant at } \mathrm{P} \leq 0.01\end{array}$ & $\begin{array}{l}\text { * Significant } \\
(+)=\text { incre }\end{array}$ & \\
\hline
\end{tabular}

Figure 1: Plasma levels of $\mathrm{GH}(\mu \mathrm{M} / \mathrm{L})$, testosterone (nM/L), cortisol ( $\mu \mathrm{g} / \mathrm{L})$, insulin $(\mu \mathrm{U} / \mathrm{L}), \mathrm{T}_{3}(\mathrm{ng} / \mathrm{dl})$ and $\mathrm{T}_{4}$ $(\mu \mathrm{g} / \mathrm{dl})$ in subjects performed moderate load (a and b) and heavy load (c and d) exercise protocol.
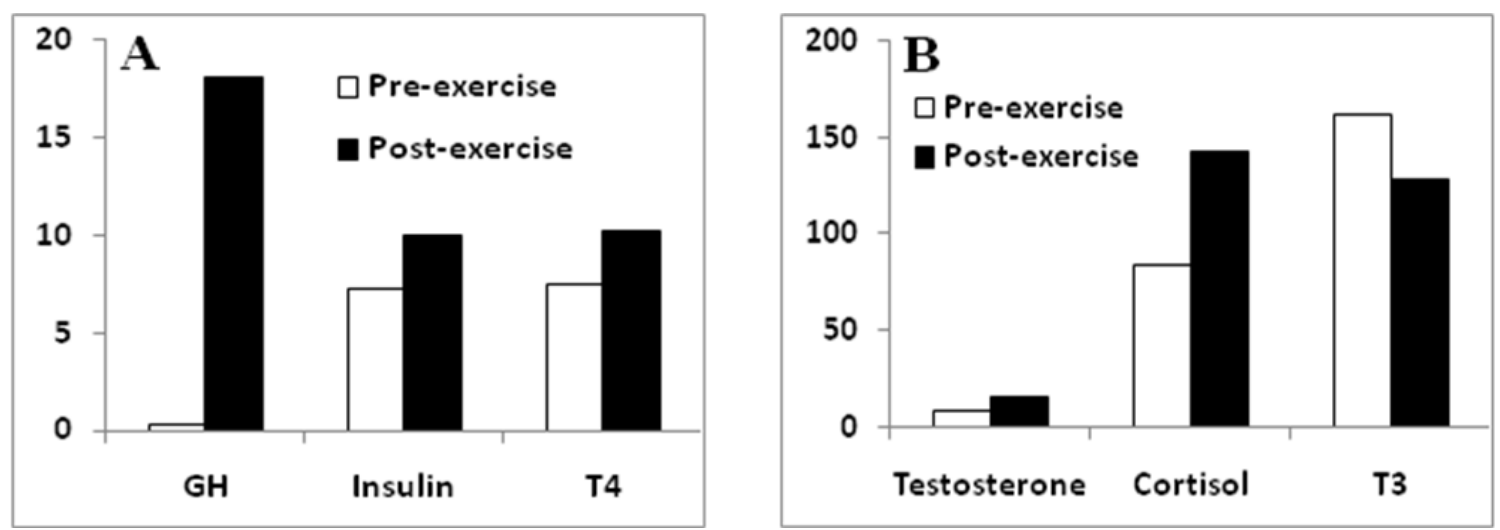



Table (3): Plasma concentrations of glucose, NEFFAs, Triglycerides (TG) and Total protein (TP) and the type of change in these parameters in subjects performed moderate load exercise protocol.

\begin{tabular}{lccc}
\hline & Pre-exercise & Post-exercise & Type of Change \\
\hline Lactate $\mathbf{~ m m o l} / \mathbf{L}$ & $3.6 \pm 0.4$ & $12.6 \pm 1.51^{* *}$ & $(+) 3.5$ fold \\
\hline Glucose $\mathbf{~ m g / d l}$ & $82 \pm 6.3$ & $67.6 \pm 3.8^{*}$ & $25(-)$ \\
\hline NEFFAs Mmol/L & $0.89 \pm 0.02$ & $1.91 \pm 0.94^{* *}$ & $115(+)$ \\
\hline Triglycerides $\mathbf{~ m g / d l}$ & $61.2 \pm 6.4$ & $132.6 \pm 8.8^{*}$ & $116(+)$ \\
\hline Total protein $\mathbf{~ g / d l}$ & $7.4 \pm 0.9$ & $8.1 \pm 1.2$ & $9(+)$ \\
\hline \multicolumn{1}{c}{ Mean \pm SE } & $*$ Significantly at $\mathrm{P} \leq 0.05$ &
\end{tabular}

** Significant at $\mathrm{P} \leq 0.01$

$(+)=$ increase $(-)$ decrease

Table (4): Plasma concentrations of glucose, NEFFAs, Triglycerides (TG) and Total protein (TP) and the type of change in these parameters in subjects performed heavy load exercise protocol.

\begin{tabular}{lccc}
\hline & Pre-exercise & Post-exercise & Type of Change \\
\hline Lactate $\mathbf{~ m M / L}$ & $3.1 \pm 0.24$ & $16.3 \pm 1.1^{* *}$ & $(+) 5.3$ fold \\
\hline Glucose $\mathbf{~ m g / d l}$ & $89 \pm 6.3$ & $71.6 \pm 3.8^{*}$ & $(-) 25$ \\
\hline NEFFA $\mu \mathbf{M} / \mathbf{L}$ & $0.81 \pm 0.02$ & $1.94 \pm 0.94^{* *}$ & $(+) 115$ \\
\hline Triglycerides $\mathbf{~ m g / d l}$ & $65.2 \pm 6.4$ & $146.1 \pm 8.8^{*}$ & $(+) 116$ \\
\hline Total protein g/dl & $7.1 \pm 0.9$ & $8.4 \pm 1.2$ & \\
\hline & Mean \pm SE & Significantly at $\mathrm{P} \leq 0.05$ & $(+)=$ increase $(-)$ decrease
\end{tabular}

Table (5): The correlation coefficient ( $\mathrm{r}$ ) between the plasma hormone levels $\left(\mathrm{T}_{3}, \mathrm{~T}_{4}\right.$, insulin and cortisol) and metabolites concentrations (glucose, NEFFAs, and triglycerides) in the subjects performed the moderate exercise load (MEL) and heavy exercise load (HEL)

\begin{tabular}{llllllll}
\hline Metabolites & \multicolumn{2}{c}{ Glucose } & \multicolumn{2}{c}{ NEFFAs } & \multicolumn{2}{c}{ Triglycerides } \\
\hline \multicolumn{1}{c}{ Hormones } & MEL & HEL & MEL & HEL & MEL & HEL \\
\hline T3 & 0.72 & 0.76 & -0.82 & -0.93 & -0.89 & -0.94 \\
\hline T4 & -0.73 & -0.79 & 0.78 & 0.86 & 0.81 & 0.92 \\
\hline Insulin & -0.88 & -0.91 & 0.91 & 0.94 & 0.84 & 0.86 \\
\hline Cortisol & -0.83 & -0.86 & 0.93 & 0.97 & 0.91 & 0.97 \\
\hline
\end{tabular}


Figure 2: Plasma concentrations of lactate $(\mathrm{mM} / \mathrm{L}$, glucose $(\mathrm{mg} / \mathrm{dl})$, FFA $(\mu \mathrm{M} / \mathrm{L})$, triglycerides $(\mathrm{TG})(\mathrm{mg} / \mathrm{dl})$ and total protein $(\mathrm{TP})(\mathrm{mg} / \mathrm{dl})$ in subjects performed moderate load (a and b) and heavy load (c and d) exercise protocol.



\section{REFERENCES}

1- Moneta, J. C., Kruk, B., Nazar, K., Krzemiñski, K., Kaciuba-U. Ci£Ko, H., Ziemba, A. (2005): Early effects of short-term endurance training on hormonal responses to graded exercise. Journal Of Physiology And Pharmacology 56, 1, 87-99.

2- Schubert, M. M., Sabapathy, S., Leveritt, M., Desbrow, B. (2014): Acute Exercise and Hormones Related to Appetite Regulation: A Meta-Analysis Sports Med. Mar; 44(3): 387-403.

3- Stensel, D. (2010): Exercise, appetite and appetiteregulating hormones: implications for food intake and weight control. Ann. Nutr. Metab. 57 Suppl 236-242.

4- Debevec, T., Amon, M., Keramidas, M. E., Kounalakis, S. N., Pisot, R., Mekjavic, I. B. (2010): Normoxic and hypoxic performance following 4 weeks of normobaric hypoxic training. Aviat Space Environ Med 81: 387-393.

5- Kayser, B., Verges, S. (2013): Hypoxia, energy balance and obesity: from pathophysiological mechanisms to new treatment strategies. Obes Rev 14: 579-592.

6- Katayama, K., Goto, K., Ishida, K., Ogita, F. (2010): Substrate utilization during exercise and recovery at moderate altitude. Metabolism 59: 959966.
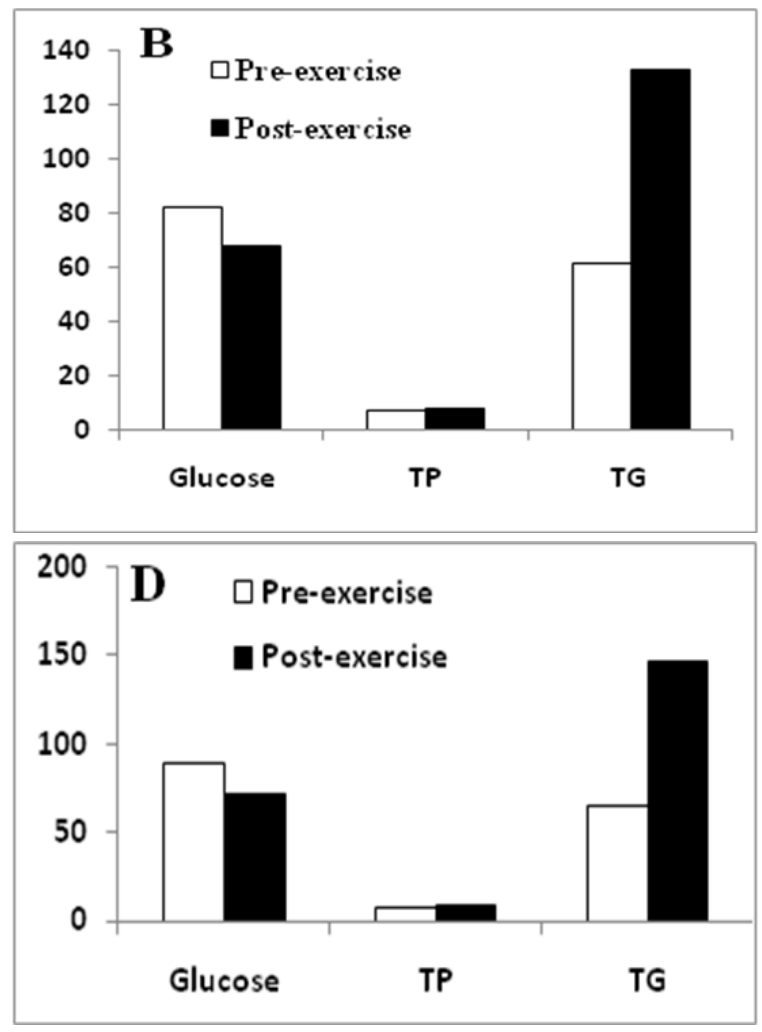

7- Fatouros, G.; Chatzinikolaou, A.; Tournis, S.; Nikolaidis, G.; Jamurtas, Z., Douroudos, I. I., Papassotiriou, I., Thomakos, P. M., Taxildaris, K., Mastorakos, G., Mitrakou, A. (2009): Intensity of Resistance Exercise Determines Adipokine and Resting Energy Expenditure Responses in Overweight Elderly Individuals. Diabetes Care. Dec 2009; 32(12): 2161-2167.

8- Enoka, R. M. (2002): Neuromechanical basis of kinesiology $\left(3^{\text {rd }}\right.$ ed.). Champaign, Illinois: Human Kinetics.

9- Hakkinen, K. (1989): Neuromuscular and hormonal adaptations during strength and power training. Journal of Sports Medicine and Physical Fitness, 29 (1), 9-26.

10-Kraemer, W. J. (1992): Influence of the endocrine system on resistance training adaptations. National Strength and Conditioning Association, 14(2), 47-54.

11-Crewther, B. T. (2004): The Mechanical, Hormonal and metabolic responses to two resistance loading schemes, Master Thesis, Health Science, Auckland University of Technology .

12-Abernethy, P. J., Jurimae, J., Logan, P. A., Taylor, A. W., Thayer, R. E. (1994): Acute and chronic responses of skeletal muscle to resistance exercise. Sports Medicine, 17(1), 22-38. 
13-Takarada, Y., Nakamura, Y., Aruga, S., Onda, T., Miyazaki, S., Ishii, N. (2000): Rapid increase in plasma growth hormone after loq-intensity resistance exercise with vascular occlusion. Journal of Applied Physiology, 88, 61-65.

14- Abraham, G. E. (1981): In clinical endocrinology, Marcel Dekker, Inc., N.Y.

15- Kosaka, K., Akauma, Y., Hogura, R., Kazuya, T. (1979): Plasma insulin response during the $100 \mathrm{~g}$ glucose tolerance test in diabetic patients.. Excerpta Med. I.C.S., 468: 283.

16- Ishikawa, T., Fujioka, H., Ishimura, T., Takenaka, A., Fujisawa, M. (2007): Ghrelin expression in human testis and serum testosterone level. Journal of Andrology 28(2):320-324

17-Abraham, G. E., Manlimos, F., Carza, R. (1977): In Handbook of RIA, G.E.Abraham ed., M. Dekker, Inc, N.Y.p. 591

18- Cooper, G., Danial, M. (1970): Standard Methods of Clinical Chemistry,vol 6,p 159.Ed RP MacDonald.New York,Academic Press, 1970.Clin. Chem. 6, 159.

19- Falholt, L., Falholt, W. (1973): An easy colorimetric micromethod for routine determination of free fatty acids in plasma. Clin Chim Acta. Jun 28;46(2):105111.

20- Wieland, D. (1974): Methods of enzymatic analysis. Vol. 3, Acad. Press. Inc.

21- Weichselbaum, T. E. (1946): Am. J. Clin. Path. Tech. Sect. 10-14.

22-Snedecor, G. W., Cochran, W. C. (1979): Statistical methods, $6^{\text {th }}$ Ed., Iowa state Univ., Press, Ames, Iowa, U.S.A.

23-Kraemer, W. J., Dziados, J. E., Marchitelli, L. J., Gordon, E. A., Harman, E. A., Mello, R., Fleck, S. J., Frykman, P. N., \& Triplett, N. T. (1993): Effect of different heavy-resistance exercise protocols on plasma beta-endorphin concentrations. Journal of Applied Physiology, 74, 450-459.

24- Burgess, M., Davis, M., Thomas, K., Buggy, J. (1993): Enrichment Resources for Laboratory Animals: Rodents. J. Appl. Physiol. 75 (2): 863-869.

25-Schwarz, E., Kindermann, W. (1990): Betaendorphin, adrenocorticotropic hormone, cortisol and catecholamines during aerobic and anaerobic exercise. Eur. J. Appl. Physiol. Occup. Physiol. 61: 165-171.

26-Hakkinen, K., Pakarinen, A. (1993): Acute hormonal responses to two different fatiguing heavy-resistance protocols in male athletes. Journal of Applied Physiology, 74, 882-887.

27- Hoffman, J. R., Kang, J. J., Maresh, C. M., Kraemer, W. J., French, D., Nioka, S., Kime, R., Rundell, K. W., Ratamess, N. A., Faigenbaum, A. D., Chance, B. (2007): Comparison of low and high-intensity resistance exercise on lipid peroxidation: role of muscle oxygenation. J. Strength Cond. Res.; 21: 118122.

28- Kraemer, W.J., and Ratamess, N.A. (2005): Hormonal responses and adaptations to resistance exercise and training. Sports Medicine, 35 (4), 339361.

29-Linnamo, V., Pakarinen, A., Komi, P.V., Kraemer, W.J., and Häkkinen, K. (2005): Acute hormonal responses to submaximal and maximal heavy resistance and explosive exercises in men and women. J. Strength Cond. Res. 19(3): 566-571.

30- Dela, F., Mikines, J., Michael, V., Galbo, H. (1992): Am. J. Physiol. 25: E1134-E1143.

31-King, S., Dalsky, P., Clutter, E., Young, M., Hollosky, O. (1988): Effects of exercise and lack of exercise on insulin sensitivity and responsiveness. J Appl Physiol 1988;64:1942-1946.

32- Ahlborg, G., Felig, P., Hagenfeldt, L., Hendler, R., Wahren, j. (1974): substrate turnover during prolonged exercise in man:splanchnic and leg metabolism of glucose, free fatty acids, and amino acids. J. Clin. Ivest. 53: 1080-1090.

33-Vagenakis, A. G., Burger, A., Portnay, G. I., Rudolph, M., O'Brain, J. T., Azizi, F., Arky, R. A., Nicod, P., Ingbar, S. H., Braverman, L. E. (1975): Diversion of Peripheral Thyroxine Metabolism from Activating to Inactivating Pathways During Complete Fasting. J. Clin. Endocrinol. Metab. 41: 1- 191.

34-O'Connell, M., Robbins, D., Horton, E., Sims, E., Danfroth, E. (1979): Changes in serum concentrations of 3,3',5'-triiodothyronine and 3,5,3'-triiodothyronine during prolonged moderate exercise. $\mathbf{J}$ Clin Endocrinol Metab. Aug;49(2):242-246.

35-Romijn, J., Klein, S., Coyle, E., Sidossis, L., Wolfe, R. (1993): Strenuous training increases lipolysis and triglyceride-fatty acid cycling at rest. J Appl Physiol 75: 108-113.

36-Knapic, J., Carol, N., Bruce, H., Evans, W. (1988): Influence of fasting on carbohydrate and fat metabolism during rest and exercise in men. J. Appl. Physiol. 64 (5): 1923-1929. 